Research Article

\title{
Separation of the Structure Signal by the Maximal Overlap Discrete Wavelet Transform and Fast Fourier Transform
}

\author{
Thanh Q. Nguyen $\mathbb{1}^{1,2}$ \\ ${ }^{1}$ Faculty of Engineering and Technology, Thu Dau Mot University, Thu Dau Mot City, Binh Duong Province, Vietnam \\ ${ }^{2}$ Thu Dau Mot University, Thu Dau Mot City, Binh Duong Province, Vietnam
}

Correspondence should be addressed to Thanh Q. Nguyen; nguyenquangthanh@tdmu.edu.vn

Received 6 May 2021; Accepted 21 July 2021; Published 4 August 2021

Academic Editor: José António Fonseca de Oliveira Correia

Copyright ( 2021 Thanh Q. Nguyen. This is an open access article distributed under the Creative Commons Attribution License, which permits unrestricted use, distribution, and reproduction in any medium, provided the original work is properly cited.

Power spectral density (PSD) is used for evaluating a structure's vibration process. Moreover, PSD not only shows a discrete form of vibration but also presents various components in a vibration structure. The superposition of multiple vibration patterns on the same spectrum poses difficulty in analyzing the spectral information in the way needed to find the structure's discrete vibration. This paper proposes a method for separating the synthetic vibration signal into a structure's discrete vibration and other extraneous vibrations using the maximal overlap discrete wavelet transform (MODWT) method combined with the method of fast Fourier transform (FFT). With the combination of these two algorithms, MODWT and FFT, the signals of the synthesized vibration have been separated into component signals with different frequency ranges. This means that PSD will be formed, which is based on the combination of the single power spectra of the component signals. Thus, the single spectrum of each of these constructed components can be used to evaluate the types of discrete vibrations coexisting in a structure's vibration process. The survey results in this paper show the sensitivity and suitability of select types of discrete vibrations to be separated out during the assessment of the structural change, so as to achieve the best possible plan for eliminating the unwanted and unexpected noise and vibration components.

\section{Introduction}

The extraction of information from the structure's vibration measurement signals is quite popular for short-term studies [1-3]. The actual vibration signals are often utilized in the time domain so as to evaluate the signal change corresponding to time. In the time domain, parameters extracted from the received signal will evaluate the changes in qualitative structure during the operation procedure $[4,5]$. Many studies have converted from the time domain to the frequency domain so as to assess the quantitative change [6-9]. This analytical technique will utilize the quantitative values of the vibration process inside the structure. The characteristics of the vibration signal are usually expressed as follows: value of the discrete vibration frequency [10-12], vibration amplitude [13], maximum power [14], damping [15], and elongation strain [16]. In the laboratory, any structure subjected to a sufficiently strong outside force will vibrate. Theoretically, the structure will take on a unique vibration corresponding to the impact of a given applied force [17]. Many vibrations are the result of the structure being subjected to an initial outside force. But many other vibrations may manifest as an effect of the accumulation of either driving and damping forces or various boundary conditions. Thus, it is necessary to eliminate these types of extraneous component vibrations, especially the noise components, in order to accurately assess the structure's most intrinsic vibration. This analysis allows for the acquisition of more signal information under the elapsed time as well as more information regarding changes in the structure itself. For the signals' component separation, many different algorithms are used, such as Fourier analysis $[18,19]$, Kalman analysis [20-22], the Gaussian noise filter analysis [23-25], and, the most widely applicable algorithm, the wavelet analysis [26-31] and many methods have been highly effective $[32,33]$. The association of wavelet analysis 
with the other algorithms improves the processing efficiency [34]. However, the wavelet studies have not yet clarified the existence and effects of the noise due to extraneous vibrations. Apart from that, the current wavelet analysis has not yet specifically indicated the relationship between the components and the overall signals. With the same point of view about research in the laboratory, experimentally observed structure vibrations result from the impact of various applied forces or from other random forces. And in the process of a structure's force bearing, the structure itself will suffer many forms of vibrations, possibly including a large amount of noise vibration. This means that the structure will simultaneously perform many types of vibrations, including both discrete and coherent vibrations, resulting in an overall oscilloscope signal with too much information. Thus, this obtained signal is not really suitable for evaluating structural changes or determining the information needed at the measured time. The synthesis resulting from many different vibrations makes the results of an assessment less accurate, and, simultaneously, the parameters utilized become less sensitive than originally expected.

With this recognition, the evaluation results will be better in the case of separating these synthetic signals into discrete signals. This paper implements the combination method of MODWT and FFT so as to analyze the signal's spectrum of actual vibration by clearly separating the characteristics of each vibration. This means that the paper still evaluates the signal change through the vibration spectrum as in previous studies, but without using the characteristics extracted from an overall vibration spectrum [35-37]. The present study separates this spectrum into discrete forms corresponding to the forms of different vibrations and simultaneously eliminates the components of noise vibration. This procedure will enhance the quality of the evaluated parameters obtained from each type of discrete vibration for the structural change. The results from this study will show the unique vibration frequency of each form of component vibration. Accordingly, these discrete vibrations can be separated according to their own natural frequencies. Furthermore, they will form a resonant area where most of the energy formed will be concentrated. Thus, the study proposes using the maximal overlap discrete wavelet transform (MODWT) on the FFT basis so as to solve the problem of identifying and separating these components. This study also identifies the signal change in both the time domain and the frequency domain. The study has separated the signal of the original vibration into component signals and simultaneously has approximated each of these signals with the signal of either noise or lack of noise. Thus, each separated signal can represent a vibrating form of the original signal corresponding to each force situation exerted on the structure. The extracted signal contains an area of vibration frequency with characteristics of its vibration conditions, which can be considered the main form of vibration. Conversely, the separated signal is not in the area of discrete vibration frequency and can be considered a form of noise. This study will help identify the structural changes over an elapsed time and sudden changes through the power spectrum. This survey will help identify the trend generated by the vibration forms embedded in the spectrum of discrete vibration power.

\section{Theoretical Basis}

2.1. Disadvantages of the Conventional Wavelet Transformation Method. Discrete wavelet transforms are considered multiresolution analysis. This analysis is applied to noise filtering and field separation during data processing [38]. In actual vibration, each vibrating signal has its own frequency response. Some types of signals, however, are extraneous and often referred to as noise signals. Generally, a signal analyzed by a wavelet transformation usually consists of two discrete components based on the area of distributed frequency: the low-frequency area and the high-frequency area. However, in many previous surveys [26-31], the high-frequency value is largely caused by noise or unwanted external environmental agents. Discrete wavelet transform is capable of decomposing the original signal into two components, the approximation and the detailed components, presented as follows:

$$
x(t)=\sum_{n} c_{j, n} \phi_{j, n}(t)+\sum_{j=J}^{\infty} \sum_{n} d_{j, n} \psi_{j, n}(t) .
$$

According to the technique of multidecomposition analysis, any $x(t)$ signals can be rewritten as the sum of signals of successive approximation and detailed components. This means that the function $x(t)$ is limited by the sum of the decomposed signals and its own successive approximation. Therefore, with the suggestion from this paper for the difference between two consecutive signals at $2^{j-1}$ and $2^{j}$, resolution is the detailed signal at $2^{j}$ resolution. The function $\phi(t)$ denotes the proportional function of two consecutive signals, and $\psi(t)$ denotes the wavelet function with the wavelet coefficient $\left\{d_{j, n}\right\}$, specified by the following equation:

$$
d_{j, n}=2^{-j / 2} \int_{-\infty}^{+\infty} x(t) \psi_{j, n}\left(2^{-j}(t-n)\right) \mathrm{d} t
$$

At the same time, the approximate function or the ratio coefficient between two consecutive signals $\left\{c_{j, n}\right\}$ is represented in the following equation:

$$
c_{j, n}=2^{-j / 2} \int_{-\infty}^{+\infty} x(t) \phi_{j, n}\left(2^{-j}(t-n)\right) \mathrm{d} t .
$$

The relationship of the ratio coefficient and the wavelet coefficient of the wavelet signal is determined through equations (4a) and (4b):

$$
\begin{aligned}
& c_{j, n}=\sum_{k \in \mathbb{Z}} g_{k} c_{j-1,2 n-k}, \\
& d_{j, n}=\sum_{k \in \mathbb{Z}} h_{k} c_{j-1,2 n-k},
\end{aligned}
$$

in which $g_{k}$ represents the filter of low-frequency area, $h_{k}$ represents the filter of high-frequency area, and $k$ is the discrete wavelet transform to the $M^{\text {th }}$ levels. Equations (4a) and $(4 \mathrm{~b})$ represent the approximate and detailed signal of the $j$ resolution level, and it is decomposed from the 
approximate signal of the $j-1$ resolution level. In the case of making the reverse analysis during the phase of signal recovery, the approximate signal segment of the $j-1$ resolution level is calculated from the approximate signal and the detailed signal at $j$ resolution through the following equation:

$$
c_{j-1, n}=\sum_{k \in \mathbb{Z}} g_{k} c_{j, 2 n-k}+\sum_{k \in \mathbb{Z}} h_{k} d_{j, 2 n-k}
$$

Equations (4a), (4b), and (5) are implemented via discrete wavelet transform (DWT). For the case in which the data are obtained for a long enough time, the method of discrete wavelet transformation is no longer correct or effective, because the amount of noise of the signal will increase when the measurement time of the signal is too long $[39,40]$. This paper proposes using MODWT instead, which is also considered an undefined discrete wavelet transformation.

\subsection{Maximal Overlap Discrete Wavelet Transform} (MODWT). The MODWT [41] is constructed by changing the size of the filters, in accordance with filter values for the given low and high-frequency areas from equations (4a) and (4b) to $\widetilde{g}_{k}=g_{k} / \sqrt{2}$ and $\widetilde{h}_{k}=h_{k} / \sqrt{2}$. Then the value of MODWT wavelet filters must meet the following properties:

$$
\begin{array}{r}
\sum_{k=0}^{K-1} \tilde{h}_{k}=0, \\
\sum_{k=0}^{K-1} \tilde{h}_{k}^{2}=\frac{1}{2}, \\
\sum_{k=-\infty}^{\infty} \tilde{h}_{k} \tilde{h}_{k+2 r}=0,
\end{array}
$$

in which $r$ is a nonzero integer and $K$ denotes the length of the wavelet wave filter. The filter will be scaled by $\left\{\widetilde{g}_{k}\right\}$ proportions with a requirement similar to $\sum_{k=0}^{K-1} \widetilde{g}_{k}=1$. The boundary condition of the signal in the time domain is $c_{0, n}^{(M)}=x_{n}(t)$. The MODWT transformation will create an approximate signal function $\left\{c_{j, n}^{(M)}\right\}$ and a detailed signal function $\left\{d_{j, n}^{(M)}\right\}$ with the $j$ resolution of the approximate signal when the resolution is $j-1$, which means $\left\{c_{j-1, n}^{(M)}\right\}$.

$$
\begin{aligned}
& d_{j-1, n}^{(M)}=\sum_{k=0}^{K-1} \tilde{h}_{k} c_{j-1,\left(n-2^{j-1} k\right) \bmod N}^{(M)}, \\
& d_{j-1, n}^{(M)}=\sum_{k=0}^{K-1} \tilde{h}_{k} c_{j-1,\left(n-2^{j-1} k\right) \bmod N}^{(M)},
\end{aligned}
$$

in which $N$ is the width of the signal sample in the time domain. Equations (7a) and (7b) can also be considered the periodic filter of the $\left\{x_{n}\right\}$ original signal using the filter, as shown in Figure 1. $\left\{\widetilde{h}_{j, k}=h_{j, k} / 2^{j / 2}\right\}$ and $\left\{\widetilde{g}_{j, k}=g_{j, k} / 2^{j / 2}\right\}$, concretely as follows:

$$
\begin{aligned}
& d_{j, n}^{(M)}=\sum_{k=0}^{K-1} \widetilde{h}_{j, k} x_{n-k \bmod N}, \\
& c_{j, n}^{(M)}=\sum_{k=0}^{K-1} \widetilde{g}_{j, k} x_{n-k \bmod N} .
\end{aligned}
$$

Figure 1 shows how the original signal can be reconstructed by $c_{j, n}^{(M)}$ and $d_{j, n}^{(M)}$ as follows:

$$
c_{j-1, n}^{(M)}=\sum_{k=0}^{K-1} \widetilde{h}_{k} d_{j,\left(n+2^{j-1} k\right) \bmod N}^{(M)}+\sum_{k=0}^{K-1} \tilde{g}_{k} c_{j,\left(n+2^{j-1} k\right) \bmod N}^{(M)},
$$

with $n=0,1,2, \ldots, N-1$. Focusing on the $j$ resolution, the average value of the MODWT wavelet coefficient $\left\{d_{j, n}^{(M)}\right\}$ is zero:

$$
E\left\{d_{j, n}^{(M)}\right\}=\sum_{k} \tilde{h}_{j, n} E\left\{x_{n-l \bmod N}\right\}=\mu_{x} \sum_{k} \tilde{h}_{j, k}=0,
$$

where $k \in Z$ and $\mu_{x}$ is the average of the signal analyzed in the time domain. And in fact, $\sum_{k} \widetilde{h}_{k}=0$ in equation (10). The attribute defined in this paper is used in wavelet-based signal change detection. Equation (10) can detect the sudden hops in the average value of the received signal. Generally, the MODWT multiresolution analysis technique presented in the paper helps to decompose an original signal into approximate and detailed fields without sampling down during such a filtering process, as well as not affecting either quantity or quality of the received signal. Therefore, the cases of decomposed data can be associated so as to reconstruct the original signal by summing the decomposed signals, as shown in Figure 2.

\subsection{The Theory for Setting up the Power Density Spectrum by} the Fast Fourier Transform. The model-built paper transforms the signal space from the time domain to the frequency domain in order to accommodate the original signal decay by the MODWT method. The original signal obtained is raw data with a lot of information incapable of being exploited if used only in the time domain. Therefore, this paper shows how signals are transferred from the time domain to the frequency domain, which will have advantages compared to utilizing them in the time domain only. Signals that are mainly utilized in the frequency domain are signals implemented through spectrum analysis, and we use the power density spectra in this study. The power density spectra needed for the Fourier transform of the signal's correlation function in the $t$ time domain at two time points $\tau$ and $T+\tau$ are found from

$$
\begin{aligned}
& \widehat{R}_{x y, \text { biased }}(m)=\frac{1}{N} \widehat{R}_{x y}(m), \\
& \text { with } \widehat{R}_{x y}(m)= \begin{cases}\sum_{n=0}^{N-m-1} x_{n+m} y_{n}^{*}, & m \geq 0, \\
\widehat{R}_{x y}^{*}(-m), & m<0,\end{cases}
\end{aligned}
$$




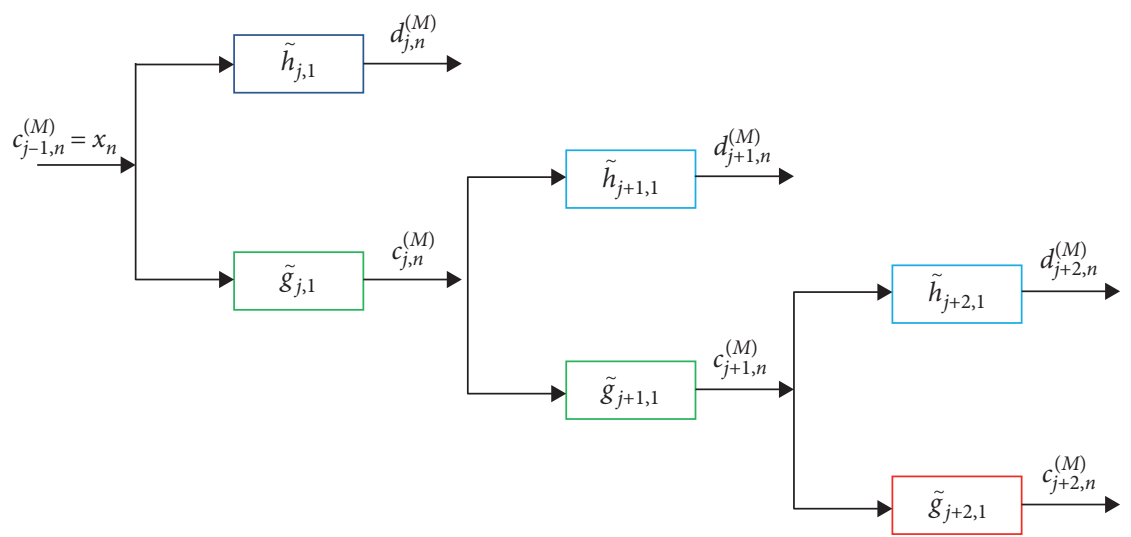

Figure 1: Wavelet separation of MODWT.

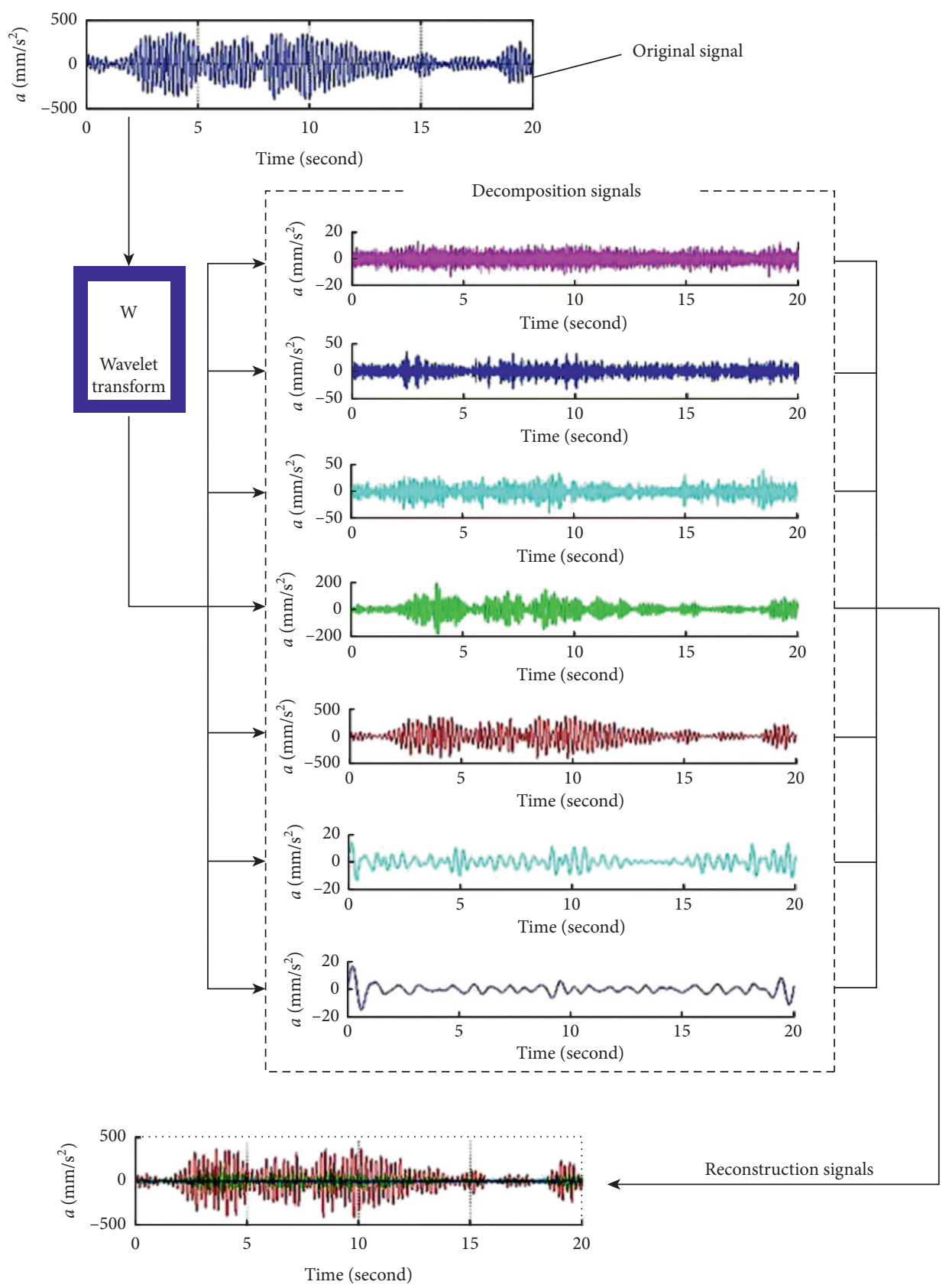

Figure 2: MODWT multiresolution analysis model [1]. 
in which $x$ is data corresponding to time $\tau$ and $y$ is data corresponding to time $T+\tau$. Thus, equation (11) allows us to correlate the two moments of the vibration signal. The correlation function will evaluate the signal randomness through the difference between the two datasets. This helps to evaluate the stability of the measured vibration signals. According to the theory, the Fourier can separate any functions into many different subcomponents, so this paper will apply the Fourier analysis method so as to separate the synthesized signal into many subcomponent signals with different corresponding frequencies. This means that the original signal in the time domain can be completely rewritten as the sum of the subfunctions generated from it. Therefore, the signal's Fourier transform $f(t) \in L^{1}(\mathbb{R})$ is usually defined by the following formula:

$$
F(\omega)=\int_{-\infty}^{+\infty} f(t) e^{-i \omega t} \mathrm{~d} t, \quad \omega \in R .
$$

If you want to make the reverse conversion from the frequency domain to the time domain, do the following according to equation

$$
f(t)=\frac{1}{2 \pi} \int_{-\infty}^{+\infty} F(\omega) e^{i \omega t} \mathrm{~d} t, \quad \omega \in R .
$$

To solve equation (13), assuming that the numbers $a$ and $b$ are large enough $(a<0, b>0)$, equation (13) is implemented as follows:

$$
F(\omega)=\int_{a}^{b} f(t) e^{-i \omega t} \mathrm{~d} t, \quad \omega \in R .
$$

Equation (15) is called a Fourier transform, and from this, the paper will generate the concept of the transformation of discrete Fourier analysis. Accordingly, we divide the segment $[a, b]$ into a sequence with $N$ elements so as to calculate the approximate equation (15):

$$
a=t_{0}<t_{1}<t_{2}<\cdots<t_{N-1}=b,
$$

$$
\text { coining } \Delta t=\frac{a-b}{N}, \quad t_{k}=a+k \Delta t, k=0,1,2, \ldots, N \text {. }
$$

Then the approximate function $\Phi(\omega)$ of $F(\omega)$ is given by the expression

$$
\Phi(\omega)=\sum_{k=0}^{N-1} f\left(t_{k}\right) e^{-i \omega t} \Delta=\sum_{k=0}^{N-1} f\left(t_{k}\right) e^{-i \omega k(b-a) / N} \Delta t,
$$

coining

$$
\omega_{n}=\frac{2 \pi n}{b-a}, \quad n=0,1,2, \ldots, N-1 .
$$

Substituting equation (18) into equation (17) gives

$$
\Phi\left(\omega_{n}\right)=e^{-i a \omega_{n}} \frac{b-a}{N} \sum_{k=0}^{N-1} f\left(t_{k}\right) e^{-i 2 \pi k(b-a) / N} .
$$

Equation (19) leads to the discrete Fourier transformation:

$$
F_{n}=F_{N}[f](n)=\sum_{k=0}^{N-1} f\left(t_{k}\right) e^{-i 2 \pi k n / N} .
$$

The FFT solver algorithm in this paper applies only to the case $N=2^{s}, s \in \mathbb{N}$. With even $N$, the sum equation (20) can be analyzed into two sums of the subcomponents, including even components of $g_{i}$ and odd components of $h_{I}$ of the $f(t)$ signal, in which the sequence $g_{j}$ is the sequence with even order, and $h_{I}$ is the sequence with odd order. The total components of each sequence have N/2 elements:

$$
\begin{aligned}
g_{i} & =f_{2 i} ; \\
h_{i} & =f_{2 i+1},\left(i=0,1,2, \ldots, \frac{N}{2}-1\right) .
\end{aligned}
$$

Applying the discrete Fourier from equation (20) to each sequence in equation (21) gives

$$
\begin{aligned}
& G_{n}=\sum_{k=0}^{(N / 2)-1} g\left(t_{k}\right) e^{-i 4 \pi k n / N}, \\
& H_{n}=\sum_{k=0}^{(N / 2)-1} h\left(t_{k}\right) e^{-i 4 \pi k n / N},
\end{aligned}
$$

coining

$$
W=e^{-i 2 \pi / N} .
$$

Substituting equation (24) into equations (20)-(22), we obtain

$$
\begin{aligned}
F_{n} & =\sum_{k=0}^{(N / 2)-1}\left[g_{k} W^{2 n k}+h_{k} W^{(2 k+1) n}\right] \\
& =\sum_{k=0}^{(N / 2)-1} g_{k} W^{2 n k}+W_{n} \sum_{k=0}^{(N / 2)-1} h_{k} W^{2 k n} F_{n}=G_{n}+W^{n} H_{n} .
\end{aligned}
$$

Thus, the $S_{n}$ value can be calculated according to the known values as $G_{n}$ and $H_{n}$. However, the above expression is only correct if

$$
n \leq \frac{N}{2}-1 .
$$

$G_{n}$ and $H_{n}$ will not be determined when $n$ is large. So for $n \geq N / 2, S_{n}$ can be calculated according to the periodic properties of the discrete Fourier transform:

$$
F_{n}=G_{n-(N / 2)}+W^{n} H_{n-(N / 2)} .
$$

We review

$$
\begin{aligned}
& W^{n}=W^{(n-(N / 2)+(N / 2))}=W^{(n-(N / 2))} W^{(N / 2),} \\
& W^{n}=e^{-i(2 \pi / 2) \cdot(N / 2)}=e^{-i \pi}=\cos \pi-i \sin \pi=-1 .
\end{aligned}
$$

From equations (28) and (29), we get 


$$
W^{n}=-W^{(n-(N / 2))}
$$

So, when $n \geq N / 2$, the Fourier transform is calculated as follows:

$$
F_{n}=G_{n-(N / 2)}-W^{n-(N / 2)} H_{n-(N / 2)} .
$$

Thus, the FFT can process the signals' autocorrelation function so as to obtain the power density spectrum in the frequency domain quicker than the conventional Fourier transformation can. We can analyze the hidden components in the signal, which would otherwise not be detected if processed in the time domain, by converting signals from the time domain to the frequency domain. The signal variation will also lead to a change in the power density spectrum.

\section{Results and Discussion}

3.1. Separating the Power Density Spectrum by the MODWT Model. The power density spectrum is a conversion of the signal vibration from the time domain to the frequency domain through the FFT transformation for the original signal's autocorrelation function. Thus, the spectrum obtained from the variation of the original signal will be a spectrum with a constant frequency. This frequency sequence may contain various discrete frequencies of distinct signal types. Accordingly, this spectrum is a combination of the power density spectra of the discrete signals that appear when being obtained from some structure. Furthermore, through analysis of maximum overlap discrete wavelets, the discrete signals and the power density spectra of these discrete signals from the typical spectrum can be separated for the purpose of representing the overall signal form, as shown in Figure 3.

It can be seen that power density spectra cover each discrete vibration area. This shows that the combination analysis between MODWT and FFT as described in this paper has helped separate the discrete signals from the total received signal. As shown in Figure 3, the signal component is decomposed at level 4 , and at this level, the signal types are almost decomposed to show the extraneous vibrations and their influence on the overall signal cluster. Therefore, this separation process also has the effect of filtering down the values of interference or unwanted signals in the overall signal. From this, we can easily find the types of signals needed to be examined, as well as signals of specific impacts. According to the signals from Figure 4, the separation study from an overall spectrum will generate three component spectra with the following characteristics: two spectra have a much larger spectral amplitude than the others. Two spectra with large amplitudes distributed into two different frequency areas include a low-frequency area with a range of $5-15 \mathrm{~Hz}$ and a high-frequency area distributed in the range of $30-50 \mathrm{~Hz}$. However, the spectrum is covered in the range $10-40 \mathrm{~Hz}$ with a very small amplitude, defined as the amount of noise caused during the measurement and data processing, or the signals are too small for us to observe and research. Thus, two spectra with large amplitudes, distributed in two different areas in the frequency sequence, are the spectral forms formed by the original signal specifically considered by us. These signals can directly affect the subjects surveyed and evaluated in this study.

Thus, the study has obtained two different types of vibration spectra for the same original signal by using the MODWT multiresolution analysis method, in which the obtained spectra have shown two different causes of impacts on the bearing structure. At the same time, the research results show that the overall PSD is only the sum of the component spectra, or, in other words, based on the component spectra, we can evaluate the structure change under each effecting agent.

\subsection{Both PSD and MODWT Model in the Actual Result.} In reality, the signal's power spectrum obtained from the structure is quite diverse, in which the spectrum is also influenced by a large amount of interference apart from the impact of the main factors on the structure. It is an issue that has received considerable interest from researchers for a long time. In addition, in practical working conditions, such as activities impacting bridges, roads, houses, and civil constructions, structures will be simultaneously affected by many different factors. These factors can be active agents, which means that various causes actively impact the building of structures according to certain purposes and clear trends. However, in addition to the factors causing direct effects, indirect factors can also significantly affect a project's operation. Figure 5 shows an experiment of a vehicle with the performance parameters as shown in Table 1, which is running through a bridge span at an approximately constant speed over the time interval being considered.

The main impact on the bridge span is the crucial impact of the wheels running along the bridge with its wheel system, as shown in Figure 5. The damping system has a direct influence during the operation and causes the vibration on the bridge under the impact of the spring system. Figure 5 shows that the vehicle creates many different vibrations during its operation that impact the structure. At positions in which the structure is in contact with the wheels, the main vibration in the mechanical system $\left(m_{1}, C_{1}\right.$, and $k_{1}$ and $m_{2}$, $C_{2}$, and $k_{2}$ ) is generated, and also generated is a series of noise signals. These types of noise signals include the amount of noise from the measurement process, the noise generated by the equipment as shown in Figure 6, and the amount of noise generated during the data processing as shown in Figure 7 . In addition, the vehicle mass $\left(m_{3}\right)$, under the impact of inertia, forms the vehicle's third vibration affecting the structure. From the two positions of the front and back wheels and the vehicle mass itself, the study has obtained the signals of vibration caused by the impact on the bridge span through an analysis of the vehicle's overall vibration impacting the bridge. If only one object influences the structure, as shown in Figure 5, the spectra obtained are as shown in Figure 8.

As shown in Figure 8, for the case in which the structure is under the impact of only one object, at different measuring positions across the overall structure the results are similar, and the comparison results are shown in Table 2. This 

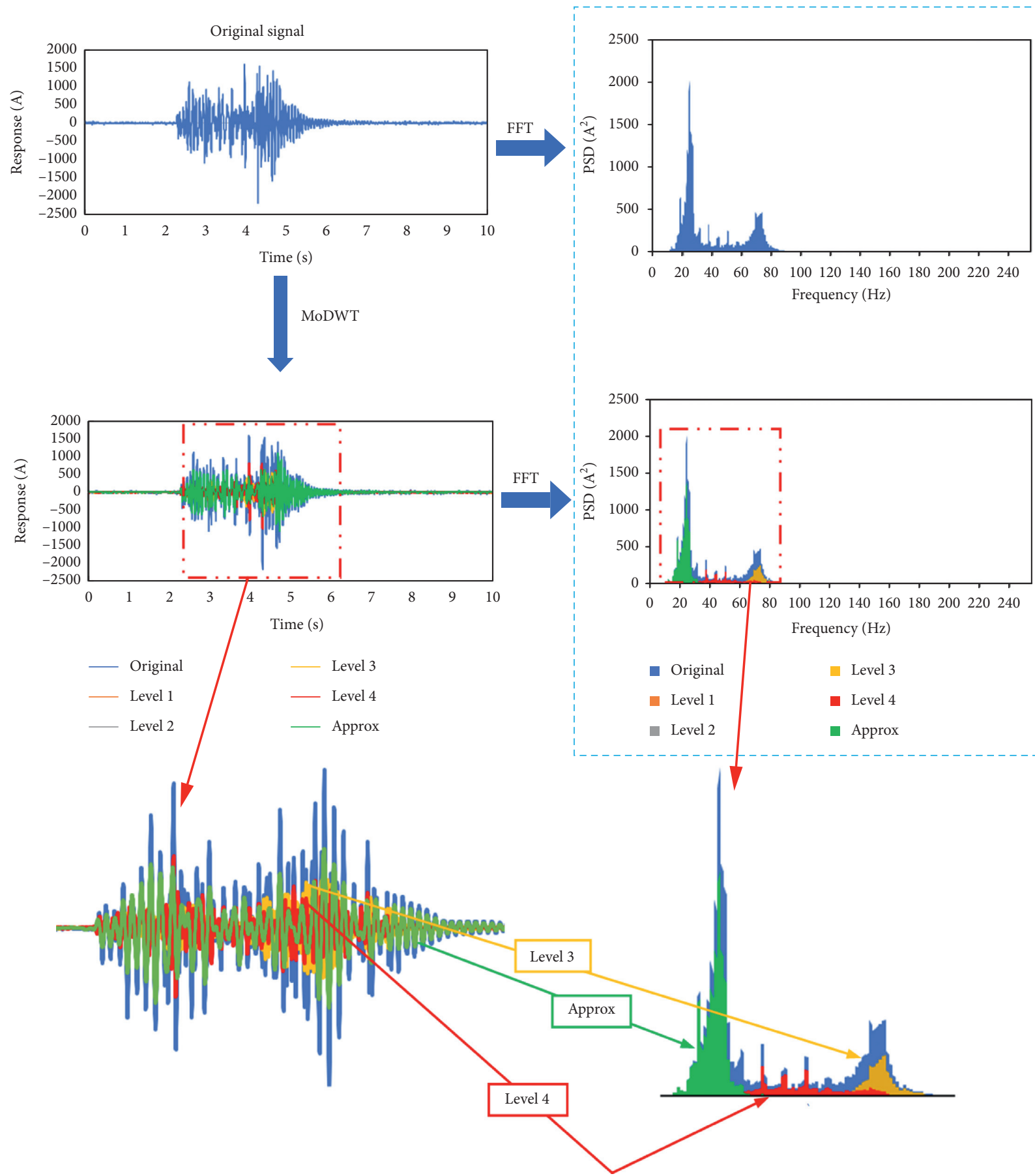

Figure 3: Spectral components analyzed by disaggregated signals.

indicates that when the impact of the object on the structure is sufficiently strong, the causative agents at close points will be similar to each other. On the contrary, the amount of noise generated at different positions is very different due to the boundary condition constraints among objects, and they are shown in Figure 8. However, although this amount of noise exists directly in the measured data, it can be eliminated during the data processing, as can be seen in the manuscript's research results, through the MODWT decomposition model. Therefore, we can completely remove the noise components, those components that do not directly affect the structure, and retain only the main impacting components through the transformation wavelet converter and power spectrum.

Taking the survey of the same object for the vehicle model moving many times on the same structure, the results are shown as Figures 9 and 10 in two cases (case 1: accelerating during movement and case 2: slowing down while moving). For both cases, the same spectrum is obtained with a value of representative power spectrum. However, the 


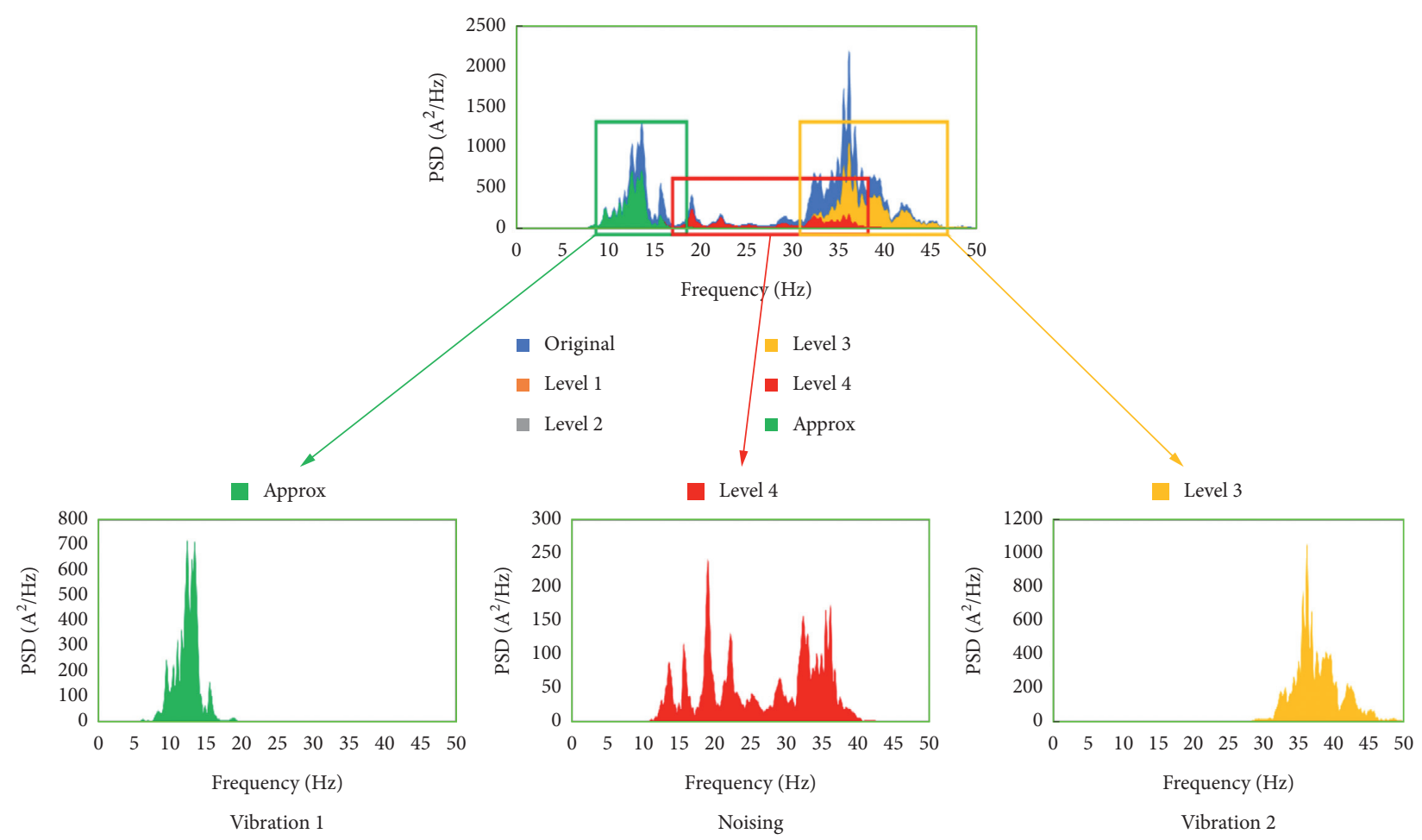

FIgURE 4: Separation of power density spectrum.

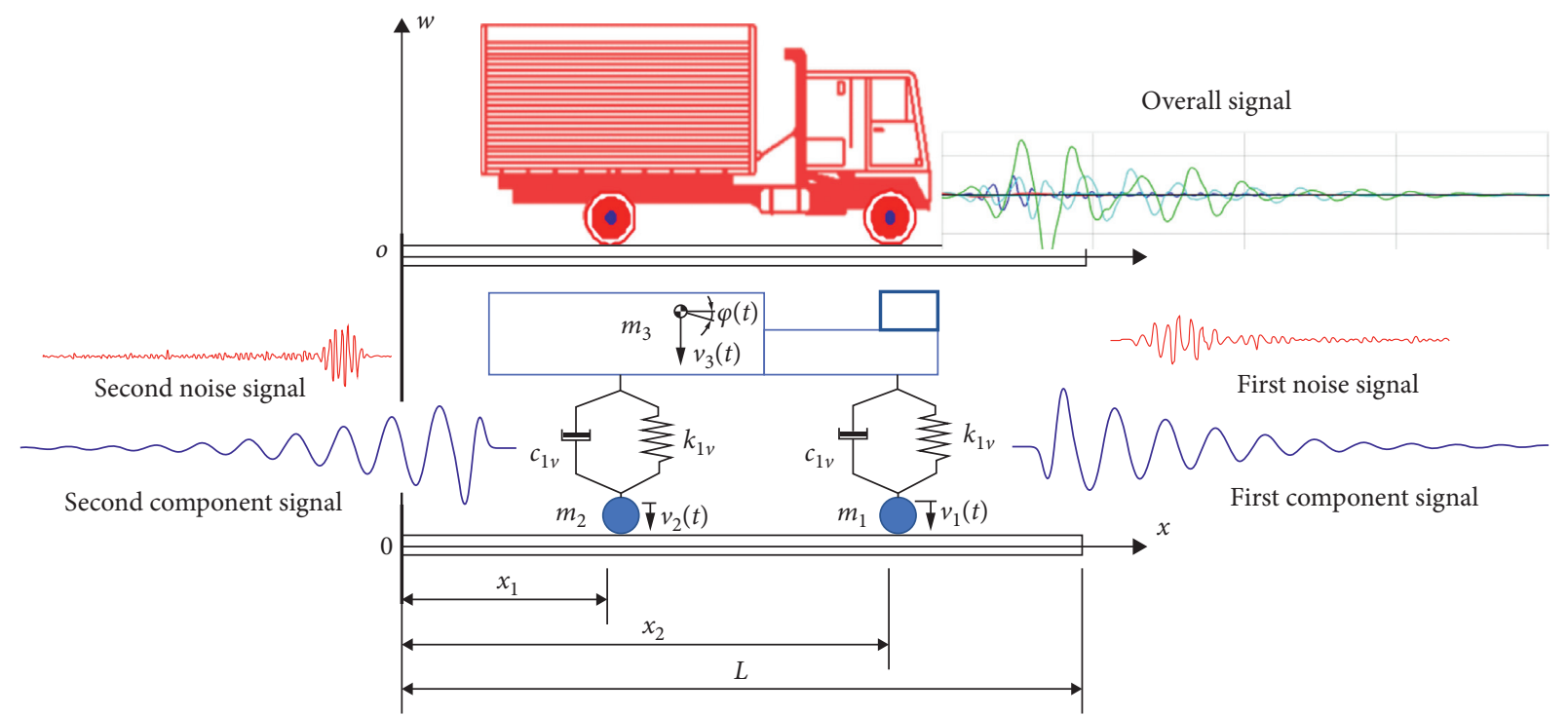

Figure 5: The established model of a vehicle on the bridge.

TABLe 1: Parameters of the vehicle model on the bridge.

Elastic modulus

Mass per unit length

Cross-sectional

Second moment of area

$4.94 \mathrm{~m}^{2}$

Shear coefficient

$0.174 \mathrm{~m}^{4}$

$5 / 6$

Beam viscous damping

$1750 \mathrm{~N} \cdot \mathrm{s} / \mathrm{m}$

Beam length

$100 \mathrm{~m}$

Mass $M_{\mathrm{eq}}$

$434.39(\mathrm{~kg})$

Spring stiffness $K_{\text {eq }}$

Damper coefficient $C_{\mathrm{eq}}$

$19097.32\left(\mathrm{~N} \cdot \mathrm{m}^{-1}\right)$

Speed $v$

$290.84\left(\mathrm{~N} \cdot \mathrm{s} \cdot \mathrm{m}^{-1}\right)$ $20\left(\mathrm{~m} \cdot \mathrm{s}^{-1}\right)$ 

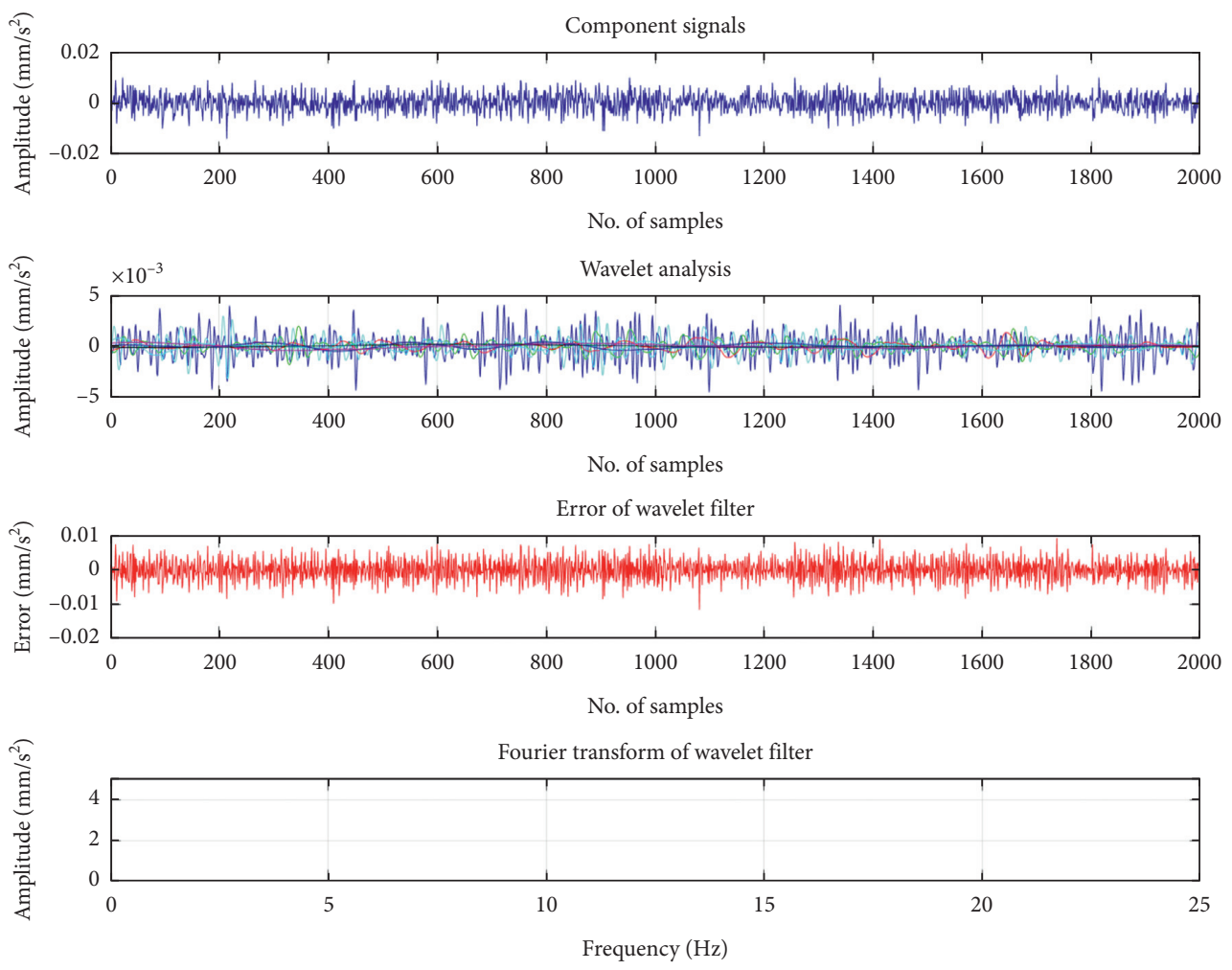

FIGURE 6: The amount of noise signal during measurement.
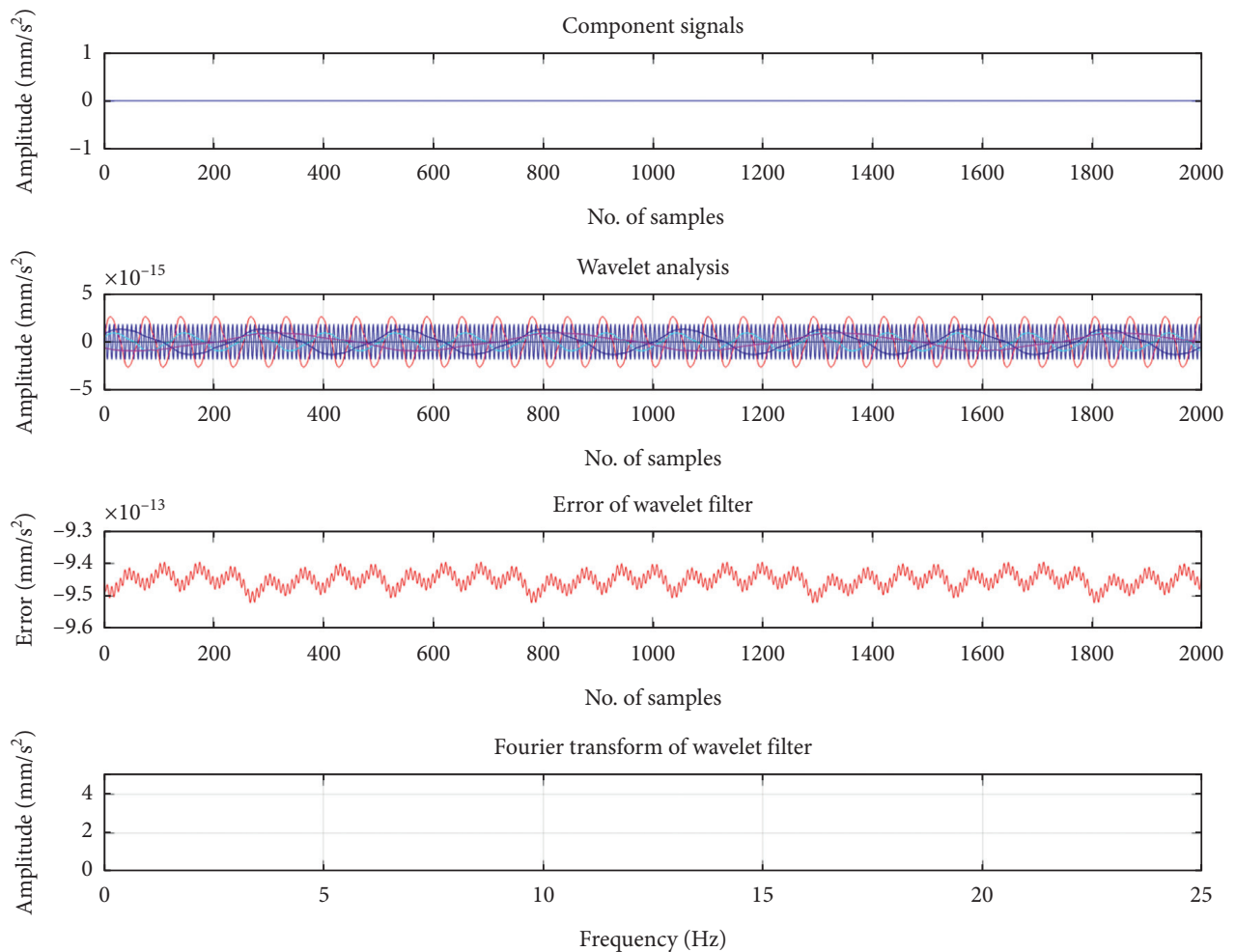

Figure 7: Amount of signal noise during data processing and use of measuring equipment.

difference in the two cases is the influence of noise, the amount of which often changes depending on the nature of the vehicle's movement.
In the first case, when the vehicle model crosses the bridge beam with a large acceleration, the noise amount abruptly changes at the end of the signal. This can be explained by the 


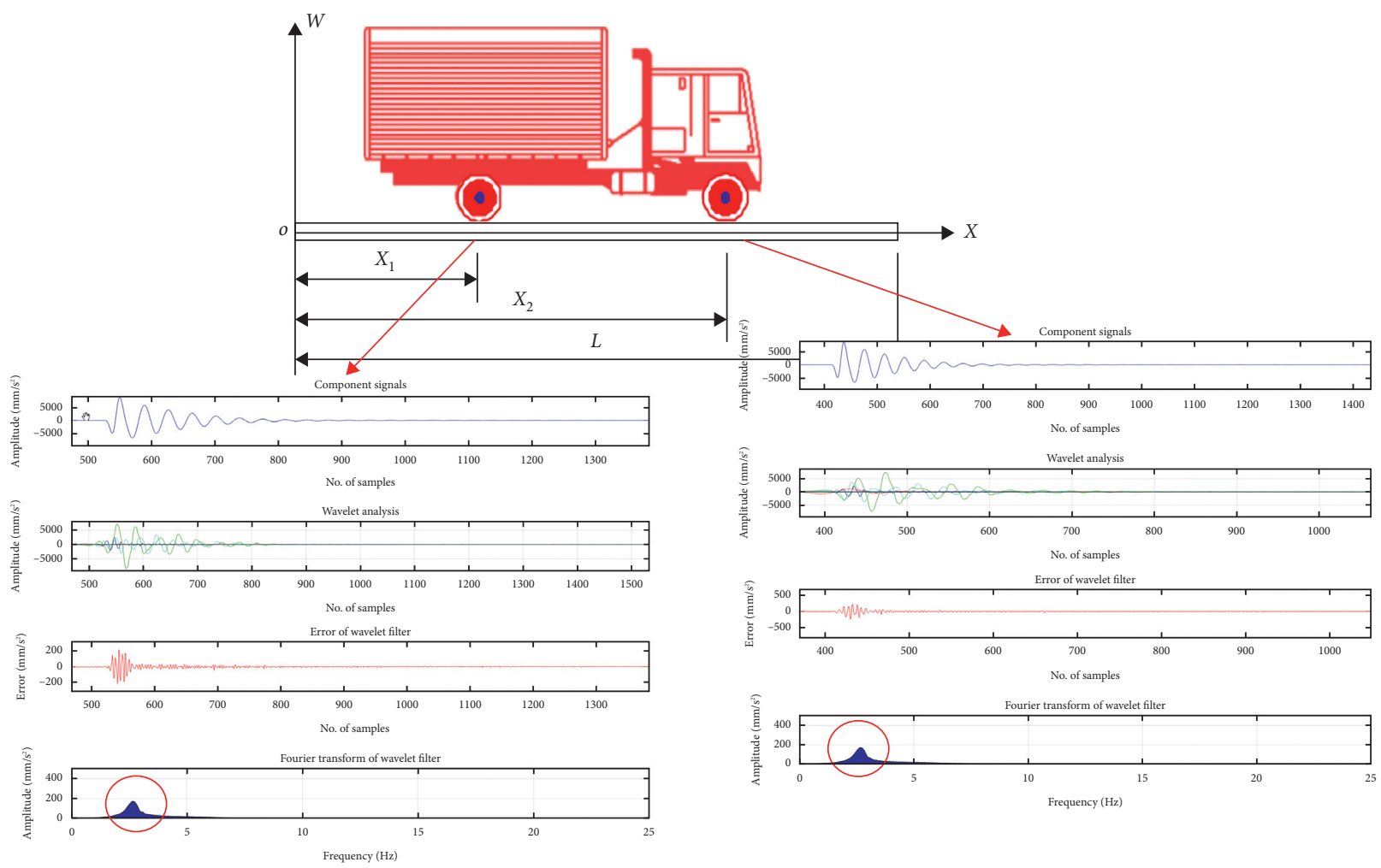

Figure 8: Vibration spectrum of the signal.

TABLE 2: Results of the PSD obtained in different status.

\begin{tabular}{|c|c|c|c|c|}
\hline Case & Parameters of the vehicle model & & $\begin{array}{c}\text { Value } \\
\text { frequencies }\end{array}$ & $\begin{array}{c}\text { Noise } \\
(\%)\end{array}$ \\
\hline \multirow{3}{*}{ Car } & $\begin{array}{l}\text { Overall dimensions: } D \times W \times H(\mathrm{~mm}) \\
4,425 \times 1,730 \times 1,475\end{array}$ & $\begin{array}{c}v=50 \mathrm{~km} / \\
\mathrm{h}\end{array}$ & $32.5(\mathrm{~Hz})$ & 10 \\
\hline & $\begin{array}{l}\text { Overall internal dimensions: }(D \times W \times C)(\mathrm{mm}) \\
\qquad 1,895 \times 1,420 \times 1,205\end{array}$ & $v=\underset{\mathrm{h}}{65 \mathrm{~km} /}$ & $33.3(\mathrm{~Hz})$ & 6.5 \\
\hline & $\begin{array}{c}\text { Wheelbase }(\mathrm{mm}) \\
\text { Wheelbase (front/rear) } 1,475 / 1,460\end{array}$ & $v=70 \mathrm{~km} /$ & $33.2(\mathrm{~Hz})$ & 6 \\
\hline \multirow{3}{*}{$\begin{array}{l}2.5 \text {-ton Hyundai mighty } \\
\text { truck }\end{array}$} & Overall dimensions: $D \times W \times H(\mathrm{~mm})$ & $\begin{array}{c}v=50 \mathrm{~km} / \\
\mathrm{h}\end{array}$ & $25.7(\mathrm{~Hz})$ & 7 \\
\hline & $\begin{array}{l}6.715 \times 2.170 \times 2.355 \\
\text { Dimensions inside the vehicle: } 4,900 \times 2,050 \times 380(\mathrm{~mm})\end{array}$ & $\begin{array}{c}v=65 \mathrm{~km} / \\
\mathrm{h}\end{array}$ & $24.3(\mathrm{~Hz})$ & 8 \\
\hline & $\begin{array}{c}\text { Wheelbase: } 3,735(\mathrm{~mm}) \\
\text { Tread Front/rear: } 1.650 / 1.495(\mathrm{~mm})\end{array}$ & $\begin{array}{c}v=70 \mathrm{~km} / \\
\mathrm{h}\end{array}$ & $24.7(\mathrm{~Hz})$ & 5 \\
\hline \multirow{4}{*}{ 5-ton truck Hyundai } & $\begin{array}{c}\text { Dimensions } D \times W \times H(\mathrm{~mm}) \\
6175 \times 2050 \times 2260\end{array}$ & $\begin{array}{l}v=50 \mathrm{~km} / \\
\mathrm{h}\end{array}$ & $12.5(\mathrm{~Hz})$ & 8 \\
\hline & $\begin{array}{l}\text { Dimensions of the vehicle } D \times R \times C(\mathrm{~mm}) \\
\qquad 4340 \times 1920 \times 390\end{array}$ & $\begin{array}{c}v=65 \mathrm{~km} / \\
\mathrm{h}\end{array}$ & $11.4(\mathrm{~Hz})$ & 6 \\
\hline & Size of ground clearance: $220(\mathrm{~mm})$ & & & \\
\hline & $\begin{array}{c}\text { Weight without load: } 3205 \mathrm{~kg} \\
\text { Gross weight: } 8440 \mathrm{~kg} \\
\text { Load capacity: } 5 \text { tons }\end{array}$ & $\begin{array}{l}v=70 \mathrm{~km} / \\
\mathrm{h}\end{array}$ & $11.6(\mathrm{~Hz})$ & 6 \\
\hline \multirow{6}{*}{ 20-ton truck } & $\begin{array}{l}\text { Iron cylinder model: ZZ1317M3861 V } \\
\text { Full allowable load (kg): } 31000\end{array}$ & $\begin{array}{l}v=30 \mathrm{~km} / \\
\mathrm{h}\end{array}$ & $8.5(\mathrm{~Hz})$ & 6 \\
\hline & $\begin{array}{l}\text { Load type }(\mathrm{kg}): 17800 \\
\text { Overall dimensions } D \times W \times H(\mathrm{~mm})\end{array}$ & $\begin{array}{c}v=40 \mathrm{~km} / \\
\mathrm{h}\end{array}$ & $7.9(\mathrm{~Hz})$ & 5 \\
\hline & $11182 \times 2496 \times 4735$ & \multirow{4}{*}{$\begin{array}{l}v=50 \mathrm{~km} / \\
\mathrm{h}\end{array}$} & \multirow{4}{*}{$7.8(\mathrm{~Hz})$} & \multirow{4}{*}{5} \\
\hline & Barrel size $D \times W \times H(\mathrm{~mm})$ & & & \\
\hline & $\begin{array}{l}10300 \times 2726 \times 800 \\
\text { Wheelbase }(\mathrm{mm}): 1800+3800+1350\end{array}$ & & & \\
\hline & Front/rear wheel track (mm): $2022(2041) / 1830$ & & & \\
\hline
\end{tabular}



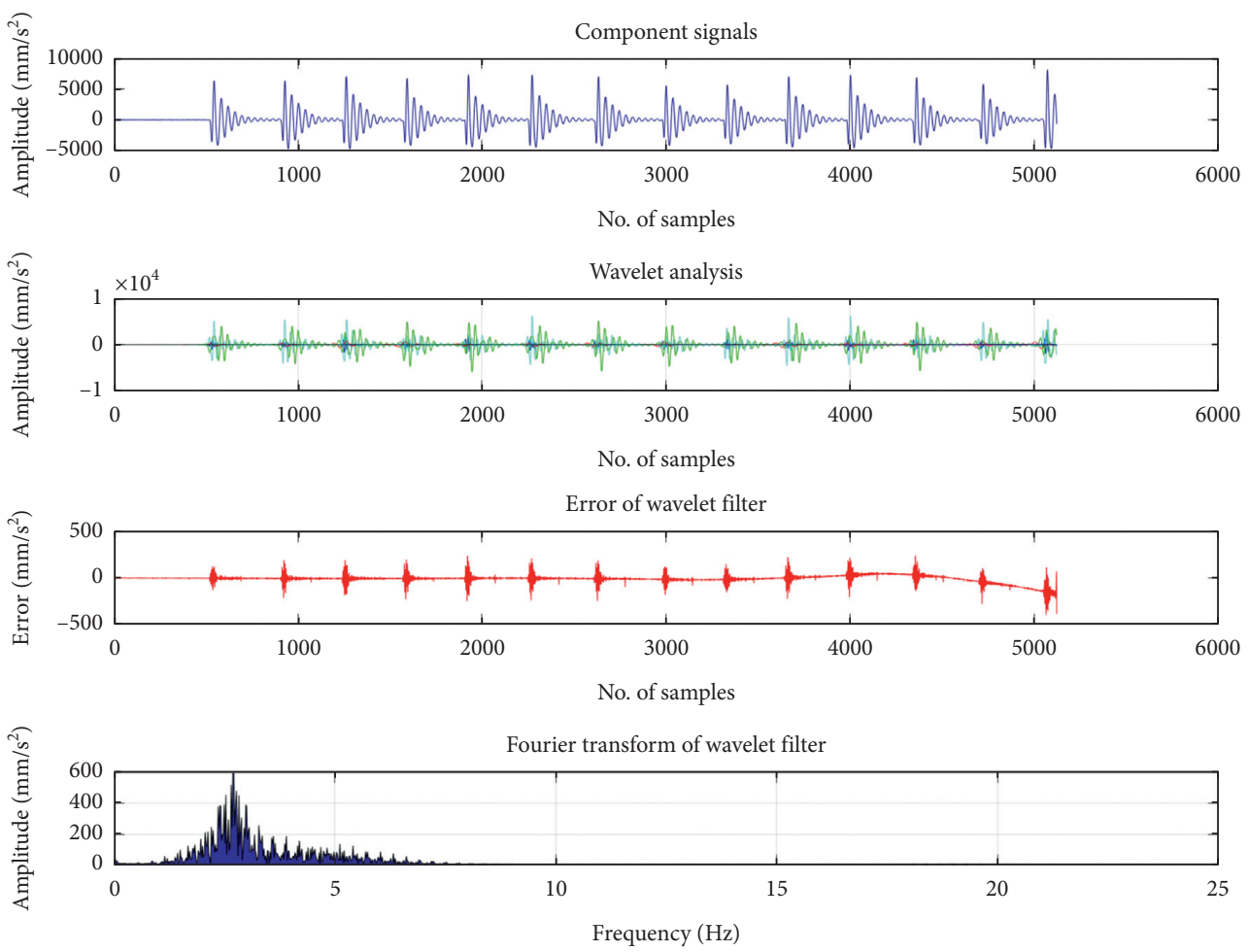

FIGURE 9: Signal of vehicle model carrying out multiple vibrations on the structure in case 1.
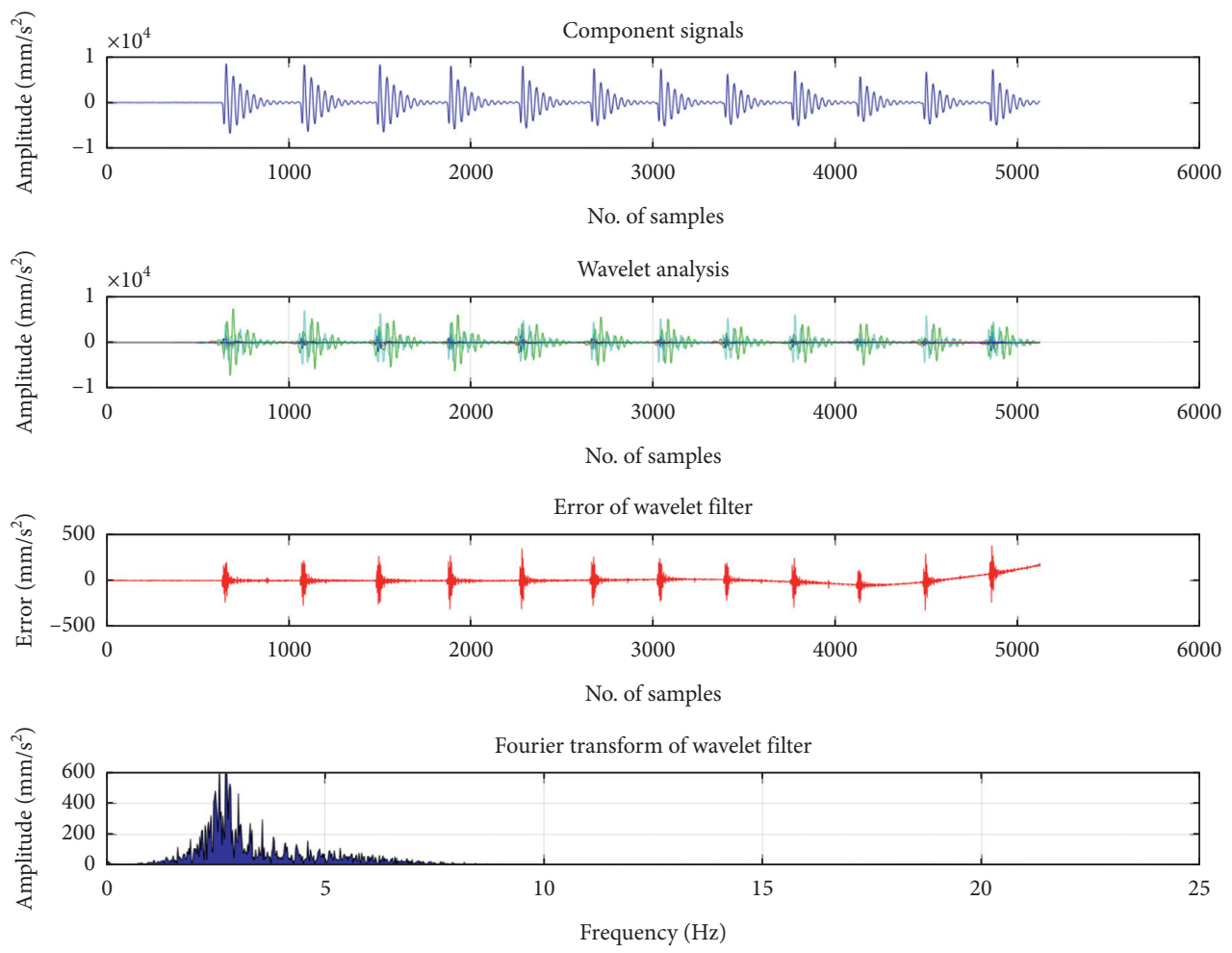

FIGURE 10: Signal of vehicle model carrying out multiple vibrations on the structure in case 2.

fact that the amount of inertia decreases during acceleration, the noise goes to the negative part of the countershaft axle, and then the structure operates in a compressed status. On the contrary, inertia increases in the case of deceleration due to the increase in noise in the positive part of the countershaft axle, which results in most of the system being under tension 


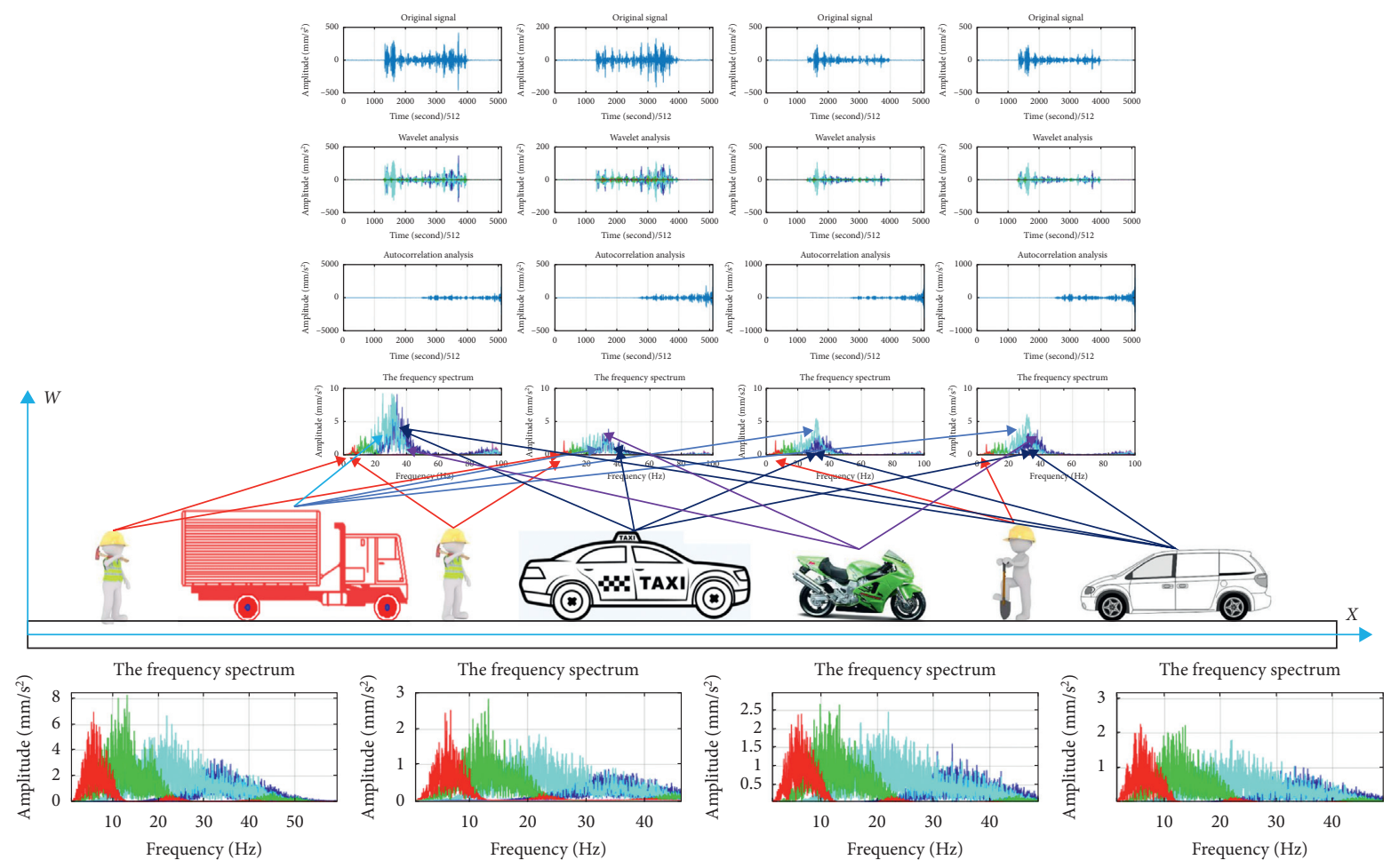

FIGURE 11: Actual signal obtained from the model.
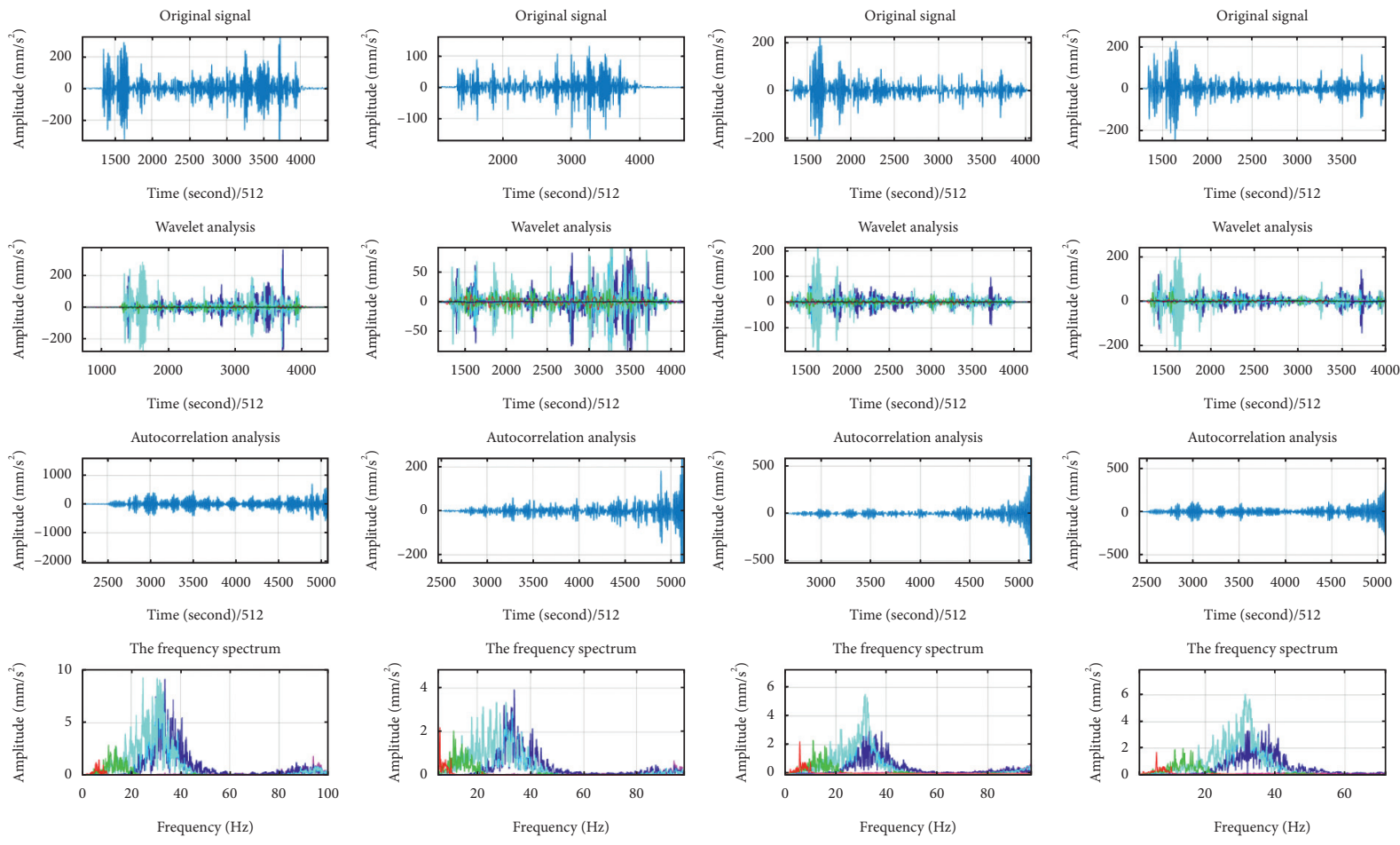

Figure 12: Actual vehicle model running on bridge in case 1.

during the operation. The amount of noise increases and becomes more complicated when putting the model into practice. Apart from the surveyed object, many other types of impacts are shown in Figure 11.
The results from Figure 11 show that each group of different objects impacting the structure will form a different type of signal. This indicates that the bridge structure is consistently influenced by many different impacts of vehicles 

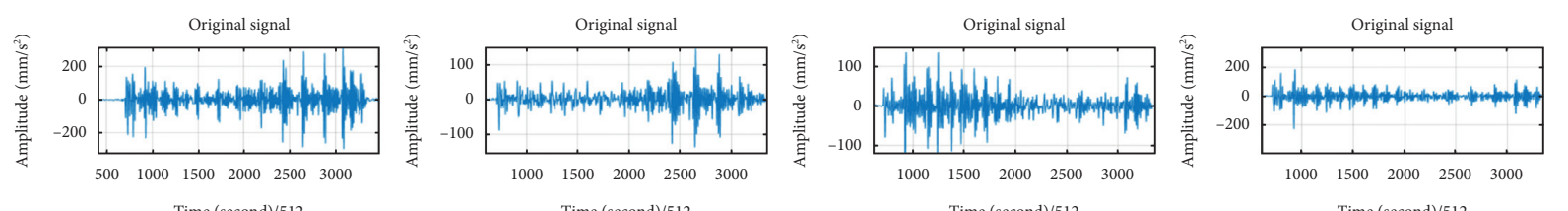

Time (second) $/ 512$
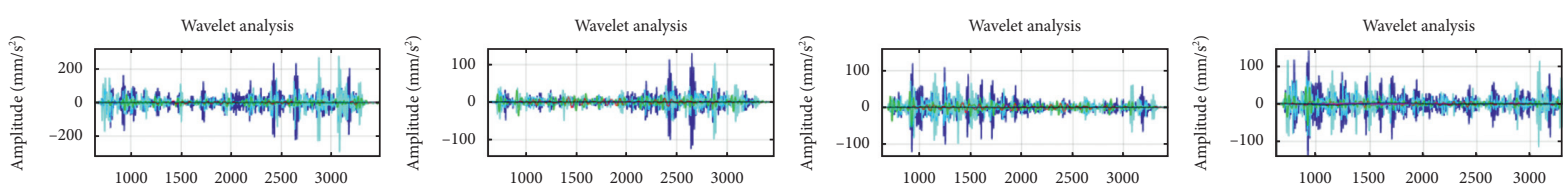

Time (second) $/ 512$

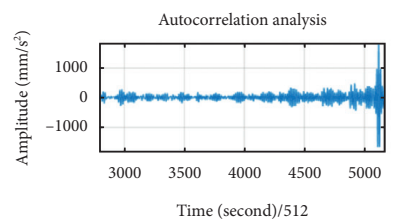

Time (second)/512
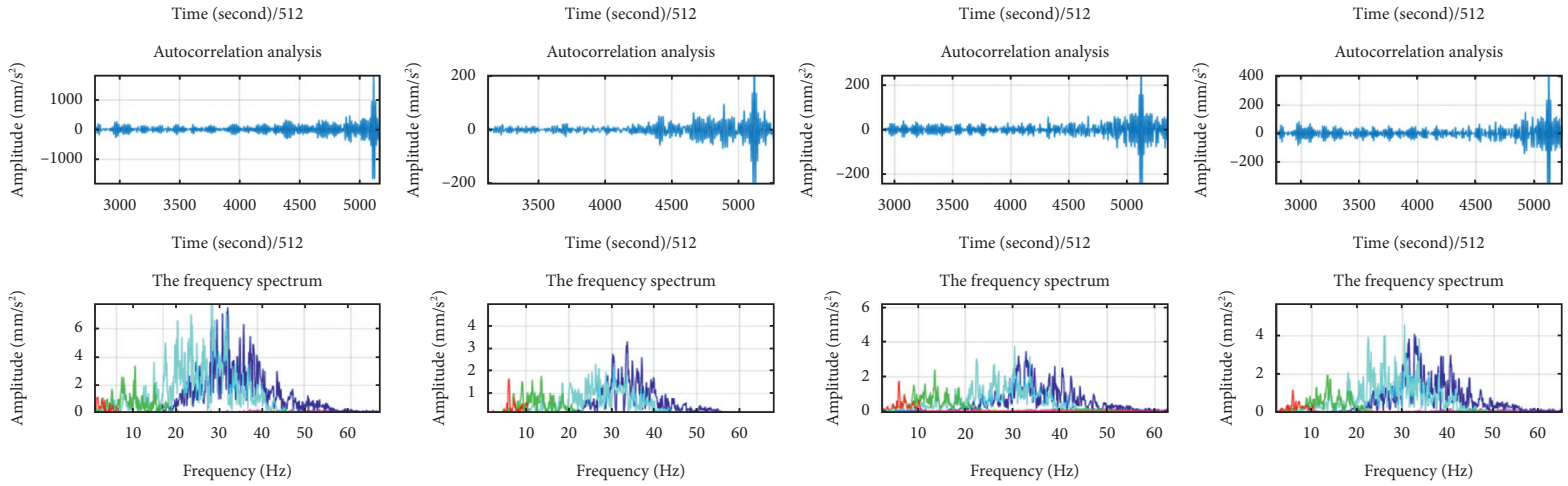

FIgURE 13: Actual vehicle model running on bridge in case 2.

and human activities. However, the cause of the structure's major vibrations must be assessed in order to accurately evaluate and forecast its lifetime as well as its operating process. For the survey above, vehicles, whether small trucks or heavier vehicles, are the main factors causing the vibrations at the bridge span structure. Small vehicles and other human activities do not affect the structure's operation but carry a considerable amount of noise in the bridge's vibration signal. Surveys of some actual models of vehicles running on bridges are shown in Figures 12 and 13. Figure 10 shows the vibration signal of the bridge span in actual circulation status with the load impacting on it being ran$\mathrm{dom}$. The second case is carried out in the status of the bridge span at rush hour when the vehicles are passing. By separating the MODWT combined with the FFT transformation method as presented in this manuscript, it can be shown that the main reciprocal impact on the bridge span is still the load amount supplied by the vehicles with significant weights. Despite being surveyed at rush hour, the spectral form divided into two cases remains nearly unchanged. This shows that, in spite of either traffic jams or crowded traffic moving across the bridge at rush hour, changes in traffic flow have little effect and the operating process remains much as usual.

\section{Conclusion}

In summary, the MODWT method combined with the transform method of FFT was used in this study to determine the actual power density spectra during signal separation. Power density spectra corresponding to discrete vibrations separated from the overall original spectrum can effectively reveal the factors causing the main impact on the structure during operating time. The process of signal separation using the technique of multiresolution analysis by the MODWT allows the capture of necessary signals and the elimination of noise signals, those signals having little effect on the structure. The main results obtained from this paper are summarized as follows:

(1) The signal of overall vibration measurement is in fact an overall signal combined with various discrete vibrations that exist as considerable noise. We can separate the original signal received into signals of discrete vibration by using the MODWT analysis technique while simultaneously eliminating the noise component in the signal. This makes the information extracted from this signal more effective with higher sensitivity than the unseparated signals.

(2) The power density spectrum obtained from the conversion of the overall vibration signal from the structure is in fact also a synthesis of the power density spectra of the discrete vibrations and the induced noise. Consequently, the survey of power spectral change will not be able to fully accommodate the signal change. Instead, we just need to examine the discrete spectra of each agent so as to find the cause of the structure change. This makes the parameters obtained from the theory presented in this paper much more sensitive than those surveyed from the overall signal.

(3) The high validity and applicability of this research have been demonstrated in this paper, showing results from the data extraction process of real structures. The study has successfully simplified the process of data extraction from the MODWT method combined with the transformation method 
of FFT. The data can be reused many times and evaluated for many different cases. The measured signals are thoroughly and effectively utilized so as to meet the demand for using big data in the world today.

\section{Data Availability}

The data used to support the findings of this study are included within the article.

\section{Disclosure}

The author confirms that the intellectual content of this paper is the original product of their work.

\section{Conflicts of Interest}

The author declares that there are no conflicts of interest.

\section{References}

[1] T. Q. Nguyen, L. C. Vuong, M. Canh Le, N. K. Ngo, and H. Nguyen- Xuan, "A data-driven approach based on wavelet analysis and deep learning for identification of multiplecracked beam structures under moving load," Measurement, vol. 162, Article ID 107862, 2020.

[2] T. D. Nguyen, T. Q. Nguyen, T. N. Nhat, H. Nguyen-Xuan, and N. K. Ngo, "A novel approach based on viscoelastic parameters for bridge health monitoring: a case study of Saigon bridge in Ho Chi Minh City Vietnam," Mechanical Systems and Signal Processing, vol. 141, Article ID 106728, 2020.

[3] T. Q. Nguyen, T. D. Nguyen, L. Q. Tran, and N. K. Ngo, “A new insight to vibration characteristics of spans under random moving load: case study of 38 bridges in Ho chi minh city," Vietnam," Shock and Vibration, vol. 2020, Article ID 1547568, 20 pages, 2020.

[4] R. Crochiere, "A weighted overlap-add method of short-time Fourier analysis/synthesis," IEEE Transactions on Acoustics, Speech, \& Signal Processing, vol. 28, no. 1, pp. 99-102, 1980.

[5] C. P. J. James and S. Saeed, "The statistical properties of raw knock signal time histories," Mechanical Systems and Signal Processing, vol. 156, Article ID 107660, 2021.

[6] T. Q. Nguyen, H. C. Doan, L. C. Vuong, H. Nguyen-Xuan, and N. K. Ngo, "Fretting fatigue damage nucleation and propagation lifetime using a central point movement of power spectral density," Shock and Vibration, vol. 2020, Article ID 4985134, 16 pages, 2020.

[7] N. W. M Bishop and F. Sherratt, "Fatigue life prediction from power spectral density data," Environmental Engineering, vol. 2, no. 1\&2, pp. 1-10, 1989.

[8] A. T. Bahill, J. S. Kallman, and J. E. Lieberman, "Frequency limitations of the two-point central difference differentiation algorithm," Biological Cybernetics, vol. 45, no. 1, pp. 1-4, 1982.

[9] T. Heida, E. C. Wentink, and E. Marani, "Power spectral density analysis of physiological, rest and action tremor in Parkinson's disease patients treated with deep brain stimulation," Journal of NeuroEngineering and Rehabilitation, vol. 10, no. 70, pp. 70-12, 2013.

[10] S. Yin, Y. Deng, T. Yu, S. Gu, and G. Zhang, "Isogeometric analysis for non-classical Bernoulli-Euler beam model incorporating microstructure and surface energy effects,"
Applied Mathematical Modelling, vol. 89, no. 1, pp. 470-485, 2021.

[11] K. Kohsaka, K. Ushijima, and W. J. Cantwellb, "Study on vibration characteristics of sandwich beam with BCC lattice core," Materials Science and Engineering: B, vol. 264, Article ID 114986, 2021.

[12] C. Lan, Z. Chen, G. Hu, Y. Liao, and W. Qin, "Achieve frequency-self-tracking energy harvesting using a passively adaptive cantilever beam," Mechanical Systems and Signal Processing, vol. 156, Article ID 107672, 2021.

[13] X.-F. Geng, Hu Ding, X.-Ye Mao, and Li-Q. Chen, "Nonlinear energy sink with limited vibration amplitude," Mechanical Systems and Signal Processing, vol. 156, Article ID 107625, 2021.

[14] E. Massara, F. Villaescusa-Navarro, S. Ho, N. Dalal, and D. N. Spergel, "Using the marked power spectrum to detect the signature of neutrinos in large-scale structure," Physical Review Letters, vol. 126, no. 1, Article ID 011301, 2021.

[15] W. Pan, H. Li, M. Wang, and L. Wang, "Elastothermodynamic damping modeling of three-layer Kirchhoff-Love microplate considering three-dimensional heat conduction," Applied Mathematical Modelling, vol. 89, pp. 1912-1931, 2021.

[16] S. Sahmani and B. Safaei, "Large-amplitude oscillations of composite conical nanoshells with in-plane heterogeneity including surface stress effect," Applied Mathematical Modelling, vol. 89, pp. 1792-1813, 2021.

[17] T. Q. Nguyen, T. T. D. Nguyen, H. Nguyen-Xuan, and N. K. Ngo, "A correlation coefficient approach for evaluation of stiffness degradation of beams under moving load," Computers, Materials \& Continua, vol. 61, no. 1, pp. 27-53, 2019.

[18] K. Stępień, W. Makieła, and S. Adamczak, "Investigating advantages and disadvantages of the analysis of a geometrical surface structure with the use of fourier and wavelet transform," Metrology and Measurement Systems, vol. 17, no. 2, pp. 233-244, 2010.

[19] M. Le, J. Kim, S. Kim, and J. Lee, "B-scan ultrasonic testing of rivets in multilayer structures based on short-time Fourier transform analysis," Measurement, vol. 128, pp. 495-503, 2018.

[20] S. C. Stubberud, R. N. Lobbia, and M. Owen, "An adaptive extended Kalman filter using artificial neural networks," in Proceedings of the 1995 34th IEEE Conference on Decision and Control, vol. 2, pp. 1852-1856, NewOrleans, LA, USA, December 1995.

[21] E. Lourens, E. Reynders, G. De Roeck, G. Degrande, and G. Lombaert, "An augmented Kalman filter for force identification in structural dynamics," Mechanical Systems and Signal Processing, vol. 27, pp. 446-460, 2012.

[22] O. Sayadi and M. Bagher Shamsollahi, "ECG denoising and compression using a modified extended kalman filter structure," IEEE Transactions on Biomedical Engineering, vol. 55, no. 9, pp. 2240-2248, 2008.

[23] K. Nair and A. Kiremidjian, "Time series based structural damage detection algorithm using Gaussian mixtures," Computer Science, Mathematics, vol. 129, no. 3, pp. 285-293, 2007.

[24] A. L. Sukstanskii and D. A. Yablonskiy, "Gaussian approximation in the theory of MR signal formation in the presence of structure-specific magnetic field inhomogeneities," Journal of Magnetic Resonance, vol. 16, pp. 236-247, 2003.

[25] F. Pascal and Y. Chitour, J.-P. Ovarlez, P. Forster, and P. Larzabal, Philippe forster and pascal larzabal, "covariance structure maximum-likelihood estimates in compound Gaussian noise: existence and algorithm analysis," IEEE 
Transactions on Signal Processing, vol. 56, no. 1, pp. 34-48, 2008.

[26] G. J. Yun, S.-G. Lee, J. Carletta, and T. Nagayama, Decentralized damage identification using wavelet signal analysis embedded on wireless smart sensors," Engineering Structures, vol. 33, no. 7, pp. 2162-2172, 2011.

[27] R. Yan and R. X. Gao, "Base wavelet selection for bearing vibration signal analysis," International Journal of Wavelets, Multiresolution and Information Processing, vol. 7, no. 4, pp. 411-426, 2009.

[28] H. Kim and H. Melhem, "Damage detection of structures by wavelet analysis," Engineering Structures, vol. 26, no. 3, pp. 347-362, 2004.

[29] A. V. Ovanesova and L. E. Suárez, "Applications of wavelet transforms to damage detection in frame structures," Engineering Structures, vol. 26, no. 1, pp. 39-49, 2004.

[30] W. Keith, I. Iakovidis, and E. J. Cross, "New results for the ADF statistic in nonstationary signal analysis with a view towards structural health monitoring," Mechanical Systems and Signal Processing, vol. 146, Article ID 106979, 2021.

[31] K. Ali and S. Rahmatalla, "Extended empirical wavelet transformation: application to structural updating," Journal of Sound and Vibration, vol. 500, Article ID 116026, 2021.

[32] Z. Wang, J. Zhou, W. Du, Y. Lei, and J. Wang, "Bearing fault diagnosis method based on adaptive maximum cyclostationarity blind deconvolution," Mechanical Systems and Signal Processing, vol. 162, Article ID 108018, 2022.

[33] Z. Wang, N. Yang, N. Li, W. Du, and J. Wang, "A new fault diagnosis method based on adaptive spectrum mode extraction," Structural Health Monitoring, 2021.

[34] D. Borras, M. Castilla, N. Moreno, and J. C. Montano, "Wavelet and neural structure: a new tool for diagnostic of power system disturbances," IEEE Transactions on Industry Applications, vol. 37, no. 1, pp. 184-190, 2001.

[35] T. Q. Nguyen and H. B. Nguyen, "Detecting and evaluating defects in beams by correlation coefficients," Shock and Vibration, vol. 2021, Article ID 6536249, 22 pages, 2021.

[36] T. Q. Nguyen, "A data-driven approach to structural health monitoring of bridge structures based on the discrete model and FFT-deep learning," Journal of Vibration Engineering \& Technologies, pp. 1-23, 2021.

[37] T. Q. Nguyen, T. D Nguyen, L. Q. Tran, and N. K Ngo, “A new insight to vibration characteristics of spans under random moving load: case study of 38 bridges in Ho chi minh city, vietnam," Shock and Vibration, vol. 2020, Article ID 1547568, 2020.

[38] R. Kamgar, M. Dadkhah, and H. Naderpour, "Seismic response evaluation of structures using discrete wavelet transform through linear analysis," Structure, vol. 29, pp. 863-882, 2021.

[39] J. Torres and S. O. Infante, "Wavelet analysis for the elimination of striping noise in satellite images," Optical Engineering, vol. 40, no. 7, pp. 1309-1314, 2001.

[40] R. Patil, "Noise reduction using wavelet transform and singular vector decomposition," Procedia Computer Science, vol. 54, pp. 849-853, 2015.

[41] H. M. S. Nawaf, F. A. Muhammad, and M. I. Nor Ashidi, "Maximal overlap discrete wavelet-packet transform aided microwave nondestructive testing," NDT \& E International, vol. 119, Article ID 102414, 2021. 